\title{
UNDESCRIBED PLANTS FROM GUATEMALA AND OTHER CENTRAL AMERICAN REPUBLICS. XXII. ${ }^{\top}$ \\ JOHN DONNELL SMITH.
}

\section{(WITH PLATE I)}

Xylosma Turrialbanum Donn. Sm.-Folia magna oblongoelliptica longe obtuseque acuminata in basin obtusiusculam aut retusam angustata membranacea integerrima glabra minute pellucido-punctata venis venulisque transversis subparallelis. Florum masculinorum pedicelli fasciculato-congesti floribus 4-5-plo longiores ad tres partes longitudinis articulati. Sepala 5. Stamina I 5 sepalis paulo breviora. Discus subinteger, glandulis nullis.

Ramis spiniformibus $\mathrm{I}-7^{\mathrm{cm}}$ longis armatum, his florum fasciculis saepe ornatis. Folia $9-\mathrm{I} 3^{\mathrm{cm}}$ longa $3-5^{\mathrm{cm}}$ lata pellucida basi nonnunquam minute bilobata, nervis lateralibus utrinque $6-8$ subtus prominulis, petiolis $5-10^{\mathrm{mm}}$ longis. Pedicelli indefiniti $9^{-\mathrm{I}} \mathrm{I}^{\mathrm{mm}}$ longi pubescentes, bracteis pilosis, bracteolis rudimentariis. Sepala oblongo-ovata $3^{\mathrm{mm}}$ longa parce pubescentia. Filamenta $2^{\mathrm{mm}}$ longa infra medium pubescentia, antheris elliptico-globosis dorso affixis. Discus annularis carnosus $2^{\mathrm{mm}}$ diametralis ciliolatus ceterum glaber. Flores feminini fructusque ignoti.- Inter species Americanas foliis integerrimis insigne.

In fundo Aragon vocato in declivibus Turrialbanis sito, Costa Rica, alt. 630 ${ }^{\mathrm{m}}$, Jan. I899, Pittier, no. 7518 Pl. Guat. \&c., qu. ed. Donn. Sm. (n. I 32 I 7 herb. nat. Cost,).

Monnina saprogena Donn. Sm. (§ Hebeandra Chodat, Bull. Herb. Boiss. 4:243.) - Folia estipulata crassiuscula glabra lanceolata-elliptica acuminata in petiolum attenuata, nervis lateralibus utrinque 3-4 erecto-patentibus distinctis ante marginem evanidis supra subimmersis, venis obsoletis. Racemi paniculati, floribus pedicellos paulo superantibus. Sepala interiora per duas partes connata. Carina subintegra. Discus conspicue unilateralis, ovario dimidiato-elliptico glabro uniloculari. Fructus

${ }^{x}$ Continued from Bot. Gaz., 27 : 443: 1899. I90I] 
inaequilateralis oblongo-ovatus acutiusculus bicarinatus et bicostatus indistincte areolatus.

Arbuscula (Tonduz in schedula), caulibus teretibus glabris. Folia $6-9^{\mathrm{cm}}$ longa $2-3^{\mathrm{cm}}$ lata, petiolis $6-7^{\mathrm{mm}}$ longis. Paniculae terminales et subterminales puberulae laxiflorae $10-16^{\mathrm{cm}}$ longae, bracteis ovatis $4^{\mathrm{mm}}$ longis ciliatis cito deciduis, pedicellis $3-4^{\mathrm{mm}}$ longis, floribus $6^{\mathrm{mm}}$ longis. Sepala pubescentia ciliataque, exteriora inferiora ovalia, superius majus ovatum $2.5^{\mathrm{mm}}$ longum, alae orbiculares $5^{\mathrm{mm}}$ diametrales extus pube punctatae e basi distincte nervosae. Carina ad apicem biplicata, petalis interioribus intus cum vagina staminea cano-pilosis, limbo lingulato $3^{\mathrm{mm}}$ longo. Stylus complanatus ovario bis longior. Fructus glaber sublaevis $6^{\mathrm{mm}}$ longus $2.5^{\mathrm{mm}}$ latus, disco gibboso glanduliformi.

In truncis putridis, La Palma, Costa Rica, alt. $1542^{\mathrm{m}}$, Aug. 1898, Tonduz, no. 7406 Pl. Guat. \&c. qu. ed. Donn. Sm. (n. 12501 herb. nat. Cost.).

Myrodia Guatemalteca Donn. Sm., Bot. Gaz. i6:2. i 89i.Hujus speciei e floribus nondum plane evolutis descriptae specimina typica cum Quararibea Guyanensi Aubl. satis congruunt. Ejusdem plantae exempla Guatemalensia nuper a Barone de Tuerckheim transmissa et flores evolutos et fructus praebent.

Heliocarpus Donnellsmithii Rose. - Mature leaves nearly orbicular, not lobed, rounded at base, shortly acuminate (tips mostly broken), glabrous and shining above, nearly glabrous below, 3 to 5-palmately veined from the base, somewhat glandular-toothed especially at base; inflorescence a large spreading panicle; sepals not appendaged; stipe slender, $5^{\mathrm{mm}}$ long; body of fruit oblong, $5^{\mathrm{mm}}$ long, somewhat hairy, becoming glabrate and rugose in age.

Near Arenal, Department Alta Verapaz, Guatemala, alt. $1500^{\mathrm{m}}$, April, I889, John Donnell Smith, n. I722 (type); Coatzacoalcos, Isthmus of Tehauntepec, State of Vera Cruz, Mexico, I 895, Charles L. Smith, n. Ioo2.

This species is very different from the numerous Mexican species, and is near $H$. Americanus Schumanni Baker, but has more glabrous leaves and an absence of hairs both on leaves and inflorescence. Mr. Baker's variety seems to me to deserve specific rank.

Explanation of Plate I. - Branch two thirds natural size; fruit $\times 3$.

Villaresia Costaricensis Donn. Sm.-Folia oblongo-elliptica utrinque praesertim apice acuminata submembranacea diaphana 


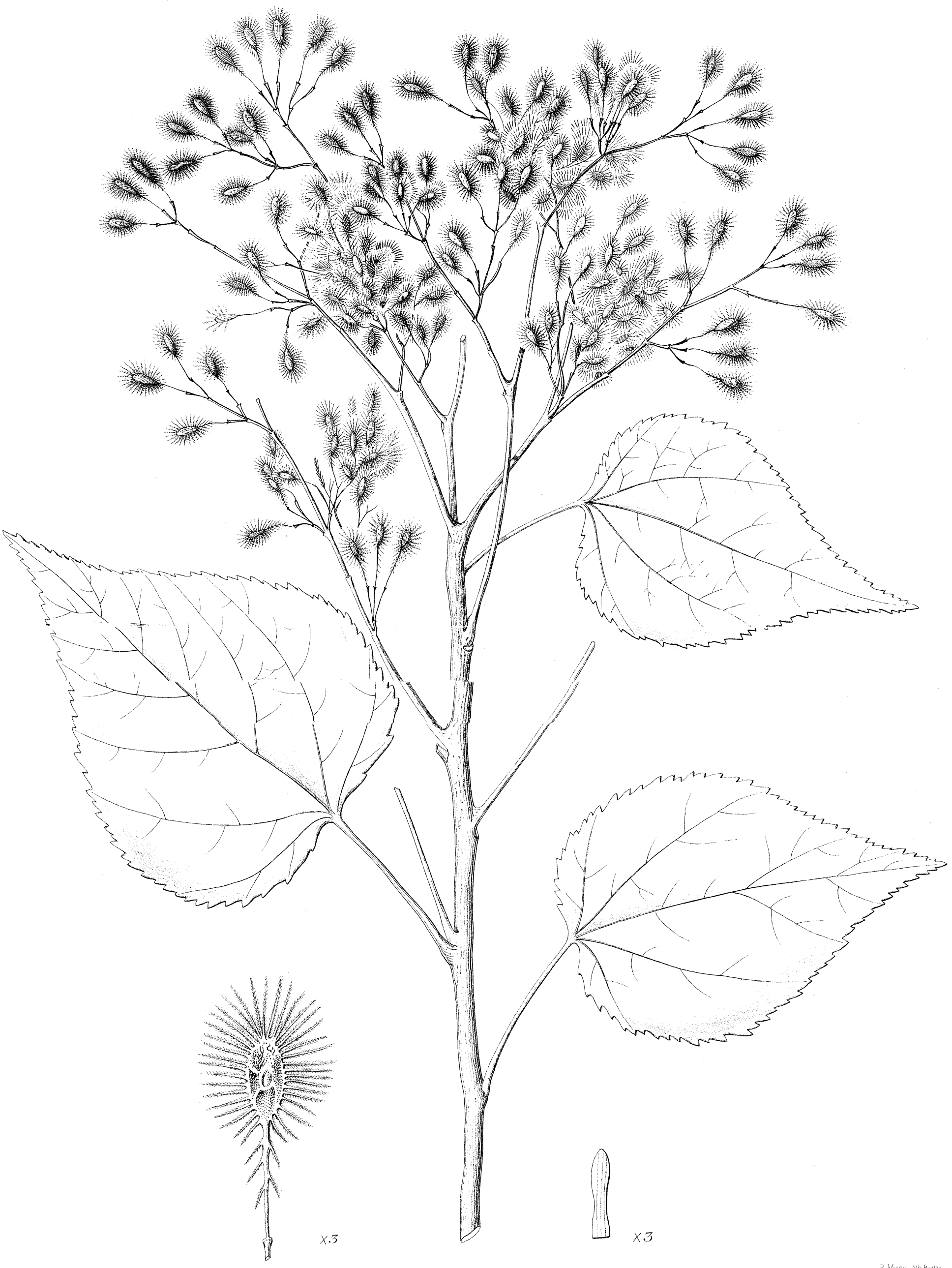


pellucido-punctulata integra, costa cum nervis lateralibus utrinque 4-5 venisque subtus conspicua. Racemi extra-axillares terminalesque foliis breviores spiciformes, glomerulis subsessilibus inferne dissitis. Sepala ovata pilosa. Petala glabra oblongoelliptica sepalis vix bis longiora. Stamina petala paene aequantia. Ovarium pilosum ovoideum I-loculare. Drupa magna olivaeformis.

Ramuli petiolique recentiores puberuli. Folia glaberrima aetate provectiore subcoriacea $9-12.5^{\mathrm{cm}}$ longa $4.5-5^{\mathrm{cm}}$ lata, petiolis crassis canaliculatis $5-7^{\mathrm{mm}}$ longis. Racemi pubescentes singuli, floriferi $3-5^{\mathrm{cm}}$ longi, fructiferi incrassati usque ad $8^{\mathrm{cm}}$ longi, floribus $3-8$ in pedicello crasso $\mathrm{I}-2^{\mathrm{mm}}$ longo sessilibus. Sepala $1.5^{\mathrm{mm}}$ longa. Petala quincuncialia apice cuspidata inflexovalvata $2.5^{\mathrm{mm}}$ longa. Stamina glabra, antheris cordato-ovalibus $\mathrm{I}^{\mathrm{mm}}$ longis. Ovarium biovulatum cum stylo brevissimo glabro adjecto petala paene aequans, stigmate obliquo nudo. Drupa pedicello vix ullo insidens $2.5^{\mathrm{cm}}$ longa $\mathrm{I} .5^{\mathrm{cm}}$ lata atque crassa apiculata in sicco nigrescens et reticulata, semisepto $5^{\mathrm{mm}}$ lato, seminis testa pallida et atro-venosa.-Genus in America cis circulum aequinoctialem adhuc non obvium.

In silvis prope Copey, Prov. Cartago, Costa Rica, alt. $1800^{\mathrm{m}}$, Febr. et Mart. I 898, Tonduz, nn. I 1664, I I 995, I 179 I herb. nat. Cost.

Blakea tuberculata Donn. Sm. (§Eublakea Triana.)-Rami crassi teretes cum petiolis pedunculis bracteis et foliorum tergo ferrugineo-furfuracei et -strigillosi. Folia ovato-orbicularia abrupte caudato-acuminata basi rotundata supra glabra. Flores fasciculati brevissime pedunculati inter maximos. Bracteae exteriores usque ad mediam connatae calycis tubum paulo superantes, interiores breviores omnino connatae. Calycis limbus ovali-cylindricus tubum aequans intus coloratus et tuberculatus, lobi retroflexi limbo bis longiores. Petala utrinque tuberculata cum staminibus basi limbi inserta. Genitalia limbo inclusa.

Folia uniuscujusque paris aequalia $\mathrm{I}_{3}^{-2} \mathrm{I}^{\mathrm{cm}}$ longa $\mathrm{I} \mathrm{O}-\mathrm{I} 5^{\mathrm{cm}}$ lata coriacea praetermisso utroque nervulo marginali trinervia, nervis in caudam oblongam $9^{\mathrm{mm}}$ longam excurrentibus, venis transversis $3-4^{\mathrm{mm}}$ inter se distantibus, petiolis robustis $4-6^{\mathrm{cm}}$ longis. Pedunculi $3-6$-fasciculati crassi $6-\mathrm{IO}^{\mathrm{mm}}$ longi. Flores diametro $6.5^{\mathrm{cm}}$, bracteis crassis opacis, exterioribus late orbicularibus $2^{\mathrm{cm}}$ longis uninerviis siccitate retroflexis et margine involutis, interioribus $\mathrm{I} .5^{\mathrm{cm}}$ longis margine crispato-plicatis. Calycis tubus campanulatus glaber, limbus $\mathrm{I}^{\mathrm{cm}}$ altus $\mathrm{I} 2^{\mathrm{mm}}$ latus carnosulus extus strigillosus in alabastro petala usque ad 
duas partes includens, lobi oblongo-ovati herbacei nervosi supra furfuracei subtus strigillosi jam ante anthesin e basi arcte retroflexi. Petala glabra oblongo-obovata $2.5-3^{\mathrm{cm}}$ longa late unguiculata carnosula rosea. Stamina in sicco rubescentia, antheris pendulis dolabriformibus acute calcaratis $8^{\mathrm{mm}}$ longis filamenta subaequantibus. Ovarium diametro $9^{\mathrm{mm}}$, centro in rostrum oblongo-conicum $5^{\mathrm{mm}}$ longum apice denticulatum producto, stylo gracili $\mathrm{I}^{\mathrm{cm}}$ longo, stigmate punctiformi. Baccae non suppetunt.- $B$. grandiflorae Hemsl. proxima.

Limbus propter exsiccationis pressionem diruptus limbum in herbario 6fissum lobis appendiculatum simulat.

Ad pascuorum margines prope La Palma, Prov. San José, Costa Rica, alt. $1550^{\mathrm{m}}$, Aug. I898, Tonduz, n. 7363 Pl. Guat., \&c., qu. ed. Donn. Sm. (n. I 2435 herb. nat. Cost.)

Anguria ovata Donn. Sm.-Tota glaberrima. Folia omnia simplicia integra membranacea concoloria ovata cuspidatoacuminata dimidio longiora quam latiora ad basin rotundam leviter retusa, nervis basilaribus 5, lateralibus utrinque 3. Flores masculini pauci subcapitati. Calyx lineari-cylindricus. Petala utrinque dense furfuracea. Antherae rectae lineares, appendice glabra.

Caulis gracilis striatus. Folia $8.5-9^{\mathrm{cm}}$ longa viridia, venulis reticulatis diaphanis, petiolis $3-5^{\mathrm{cm}}$ longis. Cirrhi tenues striati. Pedunculi masculini striati $\mathrm{I} 2-\mathrm{I} 5^{\mathrm{cm}}$ longi apice brevissime racemosi et $8-\mathrm{I} 2$-flori, pedicellis $\mathrm{I}-2^{\mathrm{mm}}$ longis. Calyx basi rotundus apice haud constrictus $14^{\mathrm{mm}}$ longus $3^{\mathrm{mm}}$ latus, dentibus obtuse ovatis $\mathrm{I}^{\mathrm{mm}}$ longis. Petala suborbicularia $6^{\mathrm{mm}}$ longa $4-5^{\mathrm{mm}}$ lata exunguiculata enervia lateritia. Antherae $\mathrm{IO}^{\mathrm{mm}}$ longae $1.5^{\mathrm{mm}}$ latae, appendice $0.5^{\mathrm{mm}}$ longa. Flores feminini fructusque desunt.-A. longipedunculatae Cogn. proxima.

In dumetis ad Las Vueltas, Tucurrique, Costa Rica, alt. $635^{\mathrm{m}}$, Feb. I 899 , Tonduz, n. I 3006 herb. nat. Cost.

Gurania Tonduziana Donn. Sm. (§F. I. Cogn. in DC. Monogr. Phan. 3: 694.) - Folia circumscriptione cordiformia paulo longiora quam latiora 5-nervia profundissime 7-partita, segmentis 5 interioribus oblanceolato-linearibus, exteriore utroque oblongo et aurito. Pedunculi masculini foliis superati, floribus brevissime spicatis. Calycis pubescentis segmenta linearia tubo 2-3-plo longiora. Antherae oblongae, appendice minuta deltoidea. 
Caulis robustus striatus cum petiolis cirrhis pedunculis pubescens. Folia $24^{\mathrm{cm}}$ longa, pagina superiore scabriuscula, inferiore pallidiore pubescente conspicue reticulato-nervosa, margine subundulata remote minuteque spinuloso-denticulata, nervo basilari utroque exteriore imum sinum marginante, segmentis acuminatis, terminali $2 \mathrm{I}^{\mathrm{cm}}$ longo ultra medium $5^{\mathrm{cm}}$ lato basi $\mathrm{I} 3^{\mathrm{mm}}$ lato ceteris interioribus paulo majore, utroque exteriore inequilaterali $6.5^{\mathrm{cm}}$ longo $3^{\mathrm{cm}}$ lato, sinibus inter segmenta rotundis a sinu basilari $2-3^{\mathrm{cm}}$ distantibus, hoc sub-rectangulari $3^{\mathrm{cm}}$ profundo $5^{\mathrm{cm}}$ lato. Pedunculi $\mathrm{I} 7^{\mathrm{cm}}$ longi, spica $8^{\mathrm{mm}}$ longa. Calycis tubus ovalis $\mathrm{I}^{\mathrm{cm}}$ longus $6^{\mathrm{mm}}$ latus, segmenta parce pubescentia $22-25^{\mathrm{mm}}$ longa basi $\mathrm{I}^{\mathrm{mm}}$ lata enervia miniata. Petala conniventia lineari-lanceolata $9-10^{\mathrm{mm}}$ longa $2^{\mathrm{mm}}$ lata enervia extus dense glandularipapillosa intus furfuracea. Antherae $7^{\mathrm{mm}}$ longae $2^{\mathrm{mm}}$ latae tertia parte replicatae, connectivo loculis angustiore, appendice vix $0.5^{\mathrm{mm}}$ longa. Flores feminini fructusque ignoti.-Ad G. Makoyanam Cogn. florum indole accedens foliis insigniter recedit.

In sylvis ad Shirores, Talamanca, Costa Rica, alt. I00 ${ }^{\mathrm{m}}, \mathrm{Feb}$. I895, Tonduz, n. 9332 herb. nat. Cost.

Sciadophyllum systylum Donn. Sm.- Ferrugineo-pubescens. Foliola 7-9 elongato-oblonga longe acutissimeque caudatoacuminata basi obtusa subcoriacea praeter costam tandem glabrescentia, nervis patulis. Umbellae in racemum simplicem cylindricum dispositae. Styli in columnam quam bacca tertia parte breviorem omnino coaliti, stigmatibus 5-6, seminibus abortu paucioribus.

Totum pube detergibili stellata interdum tomentulosa vestitum. Stipulae coriaceae lineares $5-7 \cdot 5^{\mathrm{cus}}$ longae. Petiolus teres $16-27^{\mathrm{cm}}$ longus, petiolulis canaliculatis, foliolo intimo maximo $\mathrm{I} 4-25^{\mathrm{cm}}$ longo $4-7^{\mathrm{cm}}$ lato, cauda $2.5-3 \cdot 5^{\mathrm{cm}}$ longa, petiolulo $4-7^{\mathrm{cm}}$ longo, foliolis lateralibus cum petiolulis sensim decrescentibus, infimo utroque $6-\mathrm{I} 4.5^{\mathrm{cm}}$ longo, petiolulo $2-2.5^{\mathrm{cm}}$ longo, nervis lateralibus utrinque I 2-I 8 prope marginem arcuate conjunctis. Racemi 20$35^{\mathrm{cm}}$ longi, ramis satis approximatis $1.5-4.5^{\mathrm{cm}}$ longis, pedicellis circa Io et $5-8^{\mathrm{mm}}$ longis. Baccae ovali-globosae $3-4^{\mathrm{mm}}$ longae $5-6$-angulares, juniores pube stellata punctatae, stylis $2-2.5^{\mathrm{mm}}$ longis, stigmatibus vix $0.5^{\mathrm{mm}}$ longis radiantibus, seminibus $3^{\mathrm{mm}}$ longis, $\mathrm{I}-3$ saepius abortivis. Flores ignoti.Species stylis longis totis coalitis insignis.

In sylvis ad El Alto de La Palma, Costa Rica, alt. $1542^{\mathrm{m}}$, Aug. I898, Tonduz, n. 7395 Pl. Guat. \&c., qu. ed. Donn. Sm. (n. I 2488 herb. nat. Cost.).

Oreopanax pycnocarpum Donn. Sm.-Stellato-pubescens. Folia simplicia integra crassa pube punctulata mox glabrescentia 
nitidaque ovalia utrinque rotundata triplinervia longe petiolata. Capitula fructifera spiciformia oblongo-globosa pedicellis longiora inter maxima in racemos 3-nos abbreviatos pauciramos disposita, baccis compactis stylis liberis 6-7, seminibus abortu saepius paucioribus, albumine aequabili.

Folia $\mathrm{I} 6-20^{\mathrm{cm}}$ longa $8-\mathrm{II} \mathrm{Im}^{\mathrm{cm}}$ lata, nervis subangulo angusto ascendentibus, basili utroque exteriore $8^{\mathrm{mm}}$ a basi distante, lateralibus utrinque 3 ultra medium ortis, venis transversis inter marginem et nervum utrumque basilarem subtus conspicuis circa $\mathrm{IO}$, petiolis pubescentibus $\mathrm{IO}-\mathrm{I}^{\mathrm{cm}}$ longis. Racemi pubescentes $7-9^{\mathrm{cm}}$ longi, ramis crassis $8-\mathrm{II}^{\mathrm{mm}}$ longis, inferioribus alternis, supremis 4-nis, capitulis $2.5-3.2^{\mathrm{cm}}$ longis $\mathrm{I} .8-2.2^{\mathrm{cm}}$ latis, rhachi $\mathrm{I}-\mathrm{I} .5^{\mathrm{cm}}$ longa, bracteolis cuspidato-ovatis ciliatis. Baccae subglobosae pressione mutua angulatae, nitidae in sicc. pallide flavescentes numerosissimae $8^{\mathrm{mm}}$ longae $6^{\mathrm{mm}}$ latae, stylis stellatim recurvatis $1.5^{\mathrm{mm}}$ longis, seminibus oblongis 3 -gonis $5^{\mathrm{mm}}$ longis $2^{\mathrm{mm}}$ crassis. Flores deficiunt.-Ad O. capitatum Decne. et Planch. foliis ad O. macrocephalum Seem. capitulis accedit.

In sylvis ad Copey, Prov. Cartago, Costa Rica, alt. I800 ${ }^{\mathrm{m}}$, Febr. I 898, Tonduz, n. I I 933 herb. nat. Cost.

Chomelia microloba Donn. Sm.-Inermis. Folia oblongo-ovata aut-elliptica obtuse acuminata basi acuta aut obtusa coriacea praeter nervorum axillas subtus barbatas glaberrima. Pedunculi terminales bini gracillimi, cymis bifidis multifloris, floribus ebracteolatis. Calyx glaber, ore subintegro. Corollae extus pubescentis tubus infundibuliformis calyce triplo longior, lobis induplicatovalvatis.

Ramuli recentiores cum stipulis cuspidato-triangularibus $3-4^{\mathrm{mm}}$ longis persistentibus et petiolis $4-6^{\mathrm{mm}}$ longis pubescentes. Folia $5.5-10^{\mathrm{cm}}$ longa $3-4.5^{\mathrm{cm}}$ lata, juniora e basi rotunda magis ovata, nervis lateralibus utrinque 4-5. Pedunculi glabri $2.5-3.5^{\mathrm{cm}}$ longi, cymis glabris $3.5^{\mathrm{cm}}$ latis circa $19-25-$ floris, floribus sessilibus. Calyx cylindricus $3^{\mathrm{mm}}$ longus $\mathrm{I} .5^{\mathrm{mm}}$ latus, denticulis vix $0.3^{\mathrm{mm}}$ longis obtusis. Corollae intus glabrae tubus $8-\mathrm{IO}^{\mathrm{mm}}$ longus, lobi obtuse ovati $3^{\mathrm{mm}}$ longi. Antherae semiexsertae lineares $2^{\mathrm{mm}}$ longae ad tertiam partem longitudinis affixae. Ovarium calycis duas partes aequans, stigmatibus $\mathrm{I}^{\mathrm{mm}}$ longis, ovulis linearibus $\mathrm{I}^{\mathrm{mm}}$ longis. Drupa in speciminibus suppetentibus deficit.-Ab omnibus congeneribus calyce subtruncato differt.

In sylvis litoralibus ad Santo Domingo de Golfo Dulce, Costa Rica, Febr. I 896, Tonduz, n. 7048 Pl. Guat., \&c., qu. ed. Donn. Sm. (n. 9874 herb. nat. Cost. 
Faramea trinervia K. Sch. et Donn. Sm.-Folia nitida obovato-oblonga vel oblonga contracto-acuminata auriculata subsessilia, nervo utroque basilari percurrente, lateralibus angulo subrecto a costa divergentibus. Cyma foliaceo-bibracteata trichotomo-decomposita, floribus ebracteolatis. Calycis limbus denticulatus discum aequans.

Glaberrima. Folia pergamenea diaphana laete viridia $20-25^{\mathrm{cm}}$ longa medio $6-9.5^{\mathrm{cm}}$ basi $\mathrm{I} .5^{-} 3^{\mathrm{cm}}$ lata, suprema lineari-oblonga $\mathrm{II}^{\mathrm{cm}}$ longa $3^{\mathrm{cm}}$ lata, costa utrinque prominente, nervo a basi utrinque prodeunte conspicuo apicem attingente a margine $5-10^{\mathrm{mm}}$ distante, nervis lateralibus primariis utrinque circa $\mathrm{I} 4$, nervum percurrentem attingentibus, petiolis $2-3^{\mathrm{mm}}$ longis. Stipulae $6-8^{\mathrm{mm}}$ longae in vaginam semiconnatae parte libera rotundatae aristatae. Pedunculi singuli ramulos terminantes $4-6.5^{\mathrm{cm}}$ longi apice brac teis ante anthesin caducis instructi. Cyma $4 \cdot 5-6.5^{\mathrm{cm}}$ alta, ramis late patulis complanatis, iis infimis $4-5.5^{\mathrm{cm}}$ longis, pedicellis accrescentibus $5-\mathrm{II} \mathrm{mm}$ longis. Calycis limbus ovarium aequans usque ad medium acute denticulatus. Ovarium post anthesin pedicello paulo crassius cum disco aequilongo truncato adjecto $\mathrm{I} .5^{\mathrm{mm}}$ longum. Corolla et fructus desunt.-A ceteris speciebus haec nervatione insigniter differt.

In sylvis ad Boca Zhorquin, Talamanca, Costa Rica, Mart. 1894, Tonduz, n. $857 \mathrm{I}$ herb. nat. Cost.; in sylvis Tsakianis, Talamanca, alt. $200^{\mathrm{m}}$, Apr. I895, Tonduz, n. 9583 herb. nat. Cost.

Faramea trinervia Suerrensis Donn. Sm.- Folia obovatovel oblongo-elliptica in caudam gracilem contracta infra medium in petiolum angustata, suprema lanceolata.

Frutex $3^{-4} 4^{\mathrm{m}}$ altus, ramosus. Foliorum cauda $\mathrm{I} .4-2^{\mathrm{cm}}$ longa, petioli $\mathrm{I}-\mathrm{I} .5^{\mathrm{cm}}$ longi. Bracteae lanceolatae tenuissime elongatae $6^{\mathrm{cm}}$ longae $\mathrm{I} 2^{\mathrm{mm}}$ latae. Florum nondum satis evolutorum pedicelli $2-5^{\mathrm{mm}}$ longi, calycis limbus $\mathrm{I}^{\mathrm{mm}}$ altus, dentibus triangularibus, corolla intense cyanea in sicc. nigrescens $6^{\mathrm{mm}}$ longa tubulosa usque ad mediam paene lobata, filamenta longiuscula prope basin corollae inserta, antheris $3^{\mathrm{mm}}$ longis exsertis, ovarium obovatum cum disco conico adjecto $2^{\mathrm{mm}}$ longum. Fructus cyaneus transversim depresso-ovalis $\mathrm{I}^{\mathrm{mm}}$ longus $\mathrm{II}^{\mathrm{mm}}$ crassus, semine conformi $\mathrm{II}^{\mathrm{mm}}$ longo $8^{\mathrm{mm}}$ crasso subtus usque ad medium transversim bipartito, testa pallide scariosa.

In sylvis profundis ad fundum Suerre dictum, Llanuras de Santa Clara, Comarca de Limón, Costa Rica, alt. 300 ${ }^{\mathrm{m}}$, Febr. 1896, Donn. Sm., n. 6589, Pl. Guat., \&c., qu. ed. Donn. Sm.

Parathesis glabra Donn. Sm.-Omnibus in partibus maculata et praeter corollam glabra. Folia oblongo-elliptica acuminata 
in petiolum attenuata integerrima subdiaphana utrinque dense maculata, nervis crebris patulis. Paniculae axillares foliis breviores, pedunculo filiformi, pedicellis ad apicem ramorum primariorum subumbellatis. Calycis segmenta elongato-triangularia. Corollae segmenta linearia. Antherae filamentis bis longiores prope basin affixae per totam longitudinem dehiscentes.

Folia $8-14^{\mathrm{cm}}$ longa $3.5-7^{\mathrm{cm}}$ lata maculis punctata et striatula, nervis inter se $4-6^{\mathrm{mm}}$ distantibus, petiolis canaliculatis $0.8-1.5^{\mathrm{cm}}$ longis. Paniculae cum pedunculo $2.5-4^{\mathrm{cm}}$ longo adjecto $5-7.5^{\mathrm{cm}}$ longae, ramis solitariis $7-15^{\mathrm{mm}}$ longis, pedicellis $3-6$-fasiculatis $5-7^{\mathrm{mm}}$ longis, bracteis bracteolisque lanceolatolinearibus parvis, alabastris lanceolato-conicis. Calycis segmenta $\mathrm{I}^{\mathrm{mm}}$ longa. Corollae tubus $\mathrm{I}^{\mathrm{mm}}$ altus, segmenta e basi $\mathrm{I}^{\mathrm{mm}}$ lata attenuata usque ad $7^{\mathrm{mm}}$ longa staminibus bis longiora revoluta intus praesertim marginibus sordide pubescentia. Ovarium conicum calyce brevius parce maculatum, stylo $4.5^{\mathrm{mm}}$ longo. Fructus desideratur.-Ardisiam ramifloram Oerst. habitu simulans.

In sylvis ad Copey, Prov. Cartago, Costa Rica, alt. I800 ${ }^{\mathrm{m}}, \mathrm{Febr}$. I 898, Tonduz, n. I I71 4 herb. nat. Cost.

Macroscepis congestiflora Donn. Sm., Bot. Gaz. 25: i 49. I 898. - Sub hoc nomine eandem plantam atque eodem numero indicatam incaute descripsi, quam typicam $M$. pleistanthae $l$. c . 20:543. I 895 , jam edideram.

Markea leucantha Donn. Sm.--Praeter filamenta omnibus in partibus glaberrima. Folia coriacea nitida obovato-oblonga apice rotundata a medio in petiolum attenuata. Flores apice ramorum annotinorum aggregati pauci. Calyx parvus teres coriaceus, lobis oblongo-ovatis brevissime cuspidatis. Corolla calyce 5plo longior, tubo extra calycem sensim ampliato late infundibulari. Genitalia exserta.

Frutex epiphyticus $2-3^{\mathrm{m}}$ longus verrucosus, ramulis hornotinis quadrangularibus sub apice ramorum plerumque ortis. Folia plerumque geminatim aut ternatim approximatis $7-15^{\mathrm{cm}}$ longa $2.5-5.5^{\mathrm{cm}}$ lata, recentiora magis oblanceolata et acutiuscula, nervis lateralibus utrinque 5-8 subtus tantum conspicuis, petiolis crassis $4-12^{\mathrm{mm}}$ longis. Pedicelli brevissime racemosi in speciminibus suppetentibus $3^{-8}$ circa $\mathrm{IO}^{\mathrm{mm}}$ longi, bracteis subulatis $3^{\mathrm{mm}}$ longis deciduis. Calyx intus nervosus $\mathrm{I} I-\mathrm{I} 3^{\mathrm{mm}}$ longus usque ad medium fissus. Corolla alba (Cooper in schedula) 5 -nervia reticulata $5 \cdot 3-6.3^{\mathrm{cm}}$ longa, tubo 
$3.8-4.6^{\mathrm{cm}}$ longo quam faucium latitudo bis longiore, lobis semiorbicularibus. Stamina ad $8^{\mathrm{mm}}$ supra basin corollae inserta $4-4.8^{\mathrm{cm}}$ longa, filamentis ad basin versus incano-barbatis, antheris paulo infra mediam affixis $6^{\mathrm{mm}}$ longis $2.5^{\mathrm{mm}}$ latis. Ovarium ovoideum $4^{\mathrm{mm}}$ altum atque latum, stylo $4 \cdot 3^{-5^{\mathrm{mm}}}$ longo, stigmatis obconoidei lamellis semiorbicularibus. Bacca desideratur.-Species calyce parvo insignis.

Estrella, Prov. Cartago, Costa Rica, alt. I $800^{m}$, Jun. I887, Cooper, n. 5887 Pl. Guat. \&c., qu. ed. Donn. Sm.; Prope Juan Viñas, Prov. Cartago, alt. I $140^{\mathrm{m}}$, Maj. I 89o, Tonduz, n. I 845 herb. nat. Cost.

Alloplectus macranthus Donn. Sm.--Omnibus in partibus strigilloso-pubescens. Folia maxima oblongo- vel obovatoelliptica apice acuminata in petiolum attenuata et decurrentia inaequilatera subintegra. Pedicelli racemosi bracteis breviores calycem subaequantes, floribus maximis numerosissimis coarctatis. Calycis segmenta breviter connata subaequalia oblonga obtusa integra. Corolla calyce bis longior erecta late infundibularis, limbo obliquo, lobis inaequalibus rotundatis denticulatis.

Caulis pollicis crassitudine in arborum truncis scandens. Folia $23-45^{\mathrm{cm}}$ longa $\mathrm{I} 0-\mathrm{I}_{9}{ }^{\mathrm{cm}}$ lata, nervis lateralibus utrinque $7-8$, petiolis crassis rugosis canaliculatis $4-7^{\mathrm{cm}}$ longis. Racemi aetate provectiore recurvi $2-4^{\mathrm{cm}}$ longi. Bracteae herbaceae ovales aut oblongo-ellipticae $3-4,5^{\mathrm{cm}}$ longae. Pedicelli $2.5-3^{\mathrm{cm}}$ longi, demum subsecundi. Calyx herbaceus, segmentis circa $2.5^{\mathrm{cm}}$ longis $8-\mathrm{IO}^{\mathrm{mm}}$ latis. Corolla puberula ex schedula Tuerckheimiana extus candida intus maculato-aurantiaca $6^{\mathrm{cm}}$ longa, tubo $4.2^{\mathrm{cm}}$ longo a basi saccata $6^{\mathrm{mm}}$ lata in fauces $2^{\mathrm{cm}}$ latas sensim ampliato, lobis posticis $\mathrm{I}^{\mathrm{cm}}$ anticis $7^{\mathrm{mm}}$ longis. Filamenta in vaginam brevem dilatata, antheris liberis oblongis $6^{\mathrm{mm}}$ longis. Disci glandula solitaria. Ovarium dense pubescens oblongoovatum $6^{\mathrm{mm}}$ longum, stylo $1.6^{\mathrm{mm}}$ longo, stigmate concavo. Fructus calyce aucto cinctus ovalis $\mathrm{I} .7^{\mathrm{cm}}$ longus $\mathrm{I}^{\mathrm{cm}}$ crassus, placentis rubris, seminibus oblongo-ellipticis $\mathrm{I}^{\mathrm{mm}}$ longis.-Ab $A$. circinnato Mart. foliis et inflorescentia proximo recedit praecipue corolla infundibulari altero tanto longiore..

In fundo Cubilquitz vocato, Depart. Alta Verapaz, Guatemala, alt. $35 \mathrm{o}^{\mathrm{m}}$, Jul. I 9oo, H. von Tuerckheim, n. 7642 Pl. Guat. \&c., qu. ed. Donn. Sm.Huic probabiliter referenda sunt specimina a Tonduz in Costa Rica lecta et sub n. I 3042 distributa. Haec pedicellos densissime aggregatos brevissime racemosos bracteas calycesque erubescentes praebent, corollis tamen carent.

Columnea sulfurea Donn. Sm. (§ Eucolumnea Benth. et Hook.)--Pilis aspersa. Folia disparia subtus vinosa elongatoelliptica utrinque acuminata inaequilatera basi valde obliqua. 
Pedunculi solitarii aut bini petiolo longiores calyce breviores. Calycis segmenta vinosa attenuato-linearia tubi corollini duas partes aequantia. Corolla flava infundibulari-tubulosa leviter arcuata, limbo postico quam tubus breviore quam anticus porrectus altero tanto paene longiore, galea truncata. Ovarium dense pilosum.

Epiphytica, caule crasso parum ramoso erubescente, internodiis $\mathrm{I} \cdot 5-3 \cdot 5^{\mathrm{cm}}$ longis, superioribus et petiolis pedunculis calycibus densius rubro-pilosis. Folia discoloria utrinque sparsim bulboso-pilosa ad basin latere altero obtusa altero valde exciso acuta, uniuscujusque paris folio florali $7.5-1 \mathrm{I} .5^{\mathrm{cm}}$ longo $2.5-4^{\mathrm{cm}}$ lato, minore conformi $3-4^{\mathrm{cm}}$ longo, petiolis $3-7^{\mathrm{mm}}$ longis. Pedunculi plerumque singuli $9-15^{\mathrm{mm}}$ longi. Calycis segmenta sublibera $2.5^{\mathrm{cm}}$ longa. Corolla ex sched. Tuerckheimiana sulfurea sparsim pilosa $6.8^{\mathrm{cm}}$ longa basi $3^{\mathrm{mm}}$ faucibus $7^{\mathrm{mm}}$ lata, tubo basi gibbo, limbo postico $3^{\mathrm{cm}}$ longo, galea $1.5^{\mathrm{cm}}$ longa atque lata integra, lobis lateralibus angulo recto subtriangularibus $\mathrm{I}^{\mathrm{cm}}$ longis obtusis, limbo antico lineari-oblongo $\mathrm{I} .5^{\mathrm{cm}}$ longo. Stamina $5.8^{\mathrm{cm}}$ longa, antheris oblongis $3^{\mathrm{mm}}$ longis, staminodio $4^{\mathrm{mm}}$ longo. Disci glandula solitaria crassa bidentata. Ovarium ovoideum $2^{\mathrm{mm}}$ altum, stylo rubro $6.5^{\mathrm{cm}}$ longo ad apicem versus pubescente, stigmate bilamellato. Fructus ignotus.

Cubilquitz, Depart. Alta Verapaz, Guatemala, alt. $35^{\mathrm{m}}$, Febr. I9oo, $H$. von Tuerckheim, n. 7646 Pl. Guat. \&c., qu. ed. Donn. Sm.

Columnea microcalyx macrophylla Donn. Sm.- Folia membranacea pubescentia oblongo-elliptica vix acuminata basi cordata inaequilatera, uniusque paris majus usque ad $5^{\mathrm{cm}}$ longum et $2^{\mathrm{cm}}$ latum, alterum triente minus. Calyx virescens corollae $6.7^{\mathrm{cm}}$ longae quintam partem aequans.

In sylvis prope Las Vueltas, Tucurrique, Costa Rica, alt. 650-700 ${ }^{\mathrm{m}}$, Dec. I 898, Tonduz, n. 12932 herb. nat. Cost.

Napeanthus repens Donn. Sm.- Herba repens acaulis strigilloso-pubescens. Folia opposita subrosulata petiolata tenuimembranacea oblongo-elliptica utrinque acuminata dentata. Pedicelli fasciculati filiformes. Calycis segmenta sublibera 3-nervia. Corolla rotata, tubo brevissimo, faucibus explanatis, limbo alte fisso subbilabiato. Stamina 4, loculis ovoideis divergentibus distinctis. Ovarium corollae tubum aequans. Capsula membranacea sphaerica. 
Folia $4-9^{\mathrm{cm}}$ longa $2-3 \cdot 5^{\mathrm{cm}}$ lata plerumque inaequilatera supra densius pubescentia subtus pallidiora et purpureo-reticulata, dentibus magnis crebrisque. Pedicelli complures $2-3 \cdot 5^{\mathrm{cm}}$ longi inferne marginati. Calycis segmenta lineari-lanceolata $2^{\mathrm{mm}}$ longa dense pubescentia exreticulata. Corolla alba parce pubescens diametro circa $1^{\mathrm{cm}}$, faucibus $3^{\mathrm{mm}}$ latis, segmentis parum aequalibus ovalibus $3-4^{\mathrm{mm}}$ longis. Stamina glabra, filamentis $\mathrm{I}^{\mathrm{mm}}$ longis inferne marginatis, antheris reniformibus supra medium affixis reversis $0.5^{\mathrm{mm}}$ latis ante anthesin leviter cohaerentibus, loculis poro laterali subrotundo dehiscentibus. Ovarium pubescens, stylo $3 \cdot 5^{\mathrm{mm}}$ longo, stigmate parum bifido. Capsula diametro $\mathrm{I} .5^{\mathrm{mm}}$ calyce immutato inclusa.

In rupibus praeruptis humidissimis, Cubilquitz, Depart. Alta Verapaz, Guatemala, alt. $35^{\circ}$, Jun. I 9oo, von Tuerckheim, n. 7647 P1. Guat. \&c., qu. ed. Donn. Sm.

Amphilophium oxylophium Donn. Sm.-Pilis singulis dense molliterque ochraceo-tomentosum. Stipulae foliaceae. Folia conjugata cirrho carentia, foliolis breviter petiolulatis ovato-cordiformibus contracto-acuminatis infra indumentum supra tuberculatis subtus lepidotis. Calycis limbus tubum aequans lobos 2 interiores plus quam duplo superans, lobis exterioribus triangularibus induplicatis reflexis. Corolla glabra ultra mediam labiata, labio inferiore fisso. Ovarium lepidotum glabrum.

Caulis cum petiolis thyrsoque robustus et floccoso-tomentosus. Stipulae IO-I $2^{\mathrm{mm}}$ longae deciduae. Petioli $2-2.5^{\mathrm{cm}}$ petioluli $8-\mathrm{I} 2^{\mathrm{mm}}$ metientes. Foliola $7-12^{\mathrm{cm}}$ longa $5-75^{\mathrm{cm}}$ lata supra pilis simplicibus adpressis vestita subtus pilis ramosis densius tomentosa et pallidiora. Thyrsus contracto-cylindricus in speciminibus suppetentibus vix evolutus $\mathrm{I}^{\mathrm{dm}}$ longus, bracteis bracteolisque lineari-lanceolatis $\mathrm{I}-\mathrm{I} .5^{\mathrm{cm}}$ longis. Calycis totius lepidoti tubus subglobosus $8^{\mathrm{mm}}$ longus dense pilosus, limbus utrinque parce pilosus, lobis 5 exterioribus $5^{-} 6^{\mathrm{mm}}$ longis, interioribus lati-rotundatis $3^{\mathrm{mm}}$ longis extus parce pilosis. Corolla $2.5^{\mathrm{cm}}$ longa ad apicem versus minutissime lepidota intus prope insertionem staminum linea lepidota incrassata, tubo $\mathrm{I}^{\mathrm{m}}$ longo, labii inferioris laciniis $\mathrm{I} 3^{\mathrm{mm}}$ longis. Stamina majora $\mathrm{I}^{\mathrm{mm}}$ minora $\mathrm{Io}^{\mathrm{mm}}$ metientia, thecis $2.5^{\mathrm{mm}}$ longis, staminodio $3^{\mathrm{mm}}$ longo. Discus pulvinaris cum stylo $\mathrm{I} .5^{\mathrm{cm}}$ longo glaber, stigmatibus semiorbicularibus. Capsula ignota.

Tucurrique, Costa Rica, alt. 630 ${ }^{\mathrm{m}}$, Jan. I899, Tonduz, n. I 3045 herb. nat. Cost.

Lophostachys Guatemalensis Donn. Sm.-- Folia nascentia incano-pubescentia aetate provectiore praeter petiolum nervosque 
glabrescentia disparia lanceolato-elliptica longe falcato-acuminata triente inferiore in petiolum arcuatim attenuata subtus pallidiora. Spicae terminales subsessiles solitariae, braçteis lanceolatolinearibus 5-nerviis, bracteolis spathulato-linearibus abrupte cuspidatis 2-nerviis. Calycis segmenta exteriora aequalia spathulato-oblonga cuspidato-acuminata, postico basi 7-nervio medio 5-nervio, antici usque ad medium fissi laciniis 3-nerviis. Stamina omnia perfecta.

Fruticulus decumbens dichotomo-ramosus, ramulis quadrangularibus. Folia $5-8^{\mathrm{cm}}$ longa $\mathrm{I} \cdot 5-3 \cdot 5^{\mathrm{cm}}$ lata supra saepe minutissime lineolata subtus ad costam nervosque pubescentia, petiolis pubescentibus $\mathrm{I}-\mathrm{I} .5^{\mathrm{cm}}$ longis, axillis saepius folia duo minuta producentibus. Spicae foliis reductis suffultae 3-4 $4^{\mathrm{cm}}$ longae, bracteis $9-1 \mathrm{O}^{\mathrm{mm}}$ longis $2.5-3^{\mathrm{mm}}$ latis triente superiore falcatoacuminatis et ciliatis ad nervos pubescentibus, bracteolis $9^{\mathrm{mm}}$ longis inaequilateralibus ciliatis, altera angustiore. Calyx $1.5^{\mathrm{cm}}$ longus sicut bracteolae erubescens et pulchre reticulatus ciliatus ad basin versus utrinque incanopubescens ceterum minute puberulus, segmentis lateralibus subulatis $8^{\mathrm{mm}}$ longis, postico $5^{\mathrm{mm}}$ lato. Corolla purpurea (ut videtur), pubescens $3 \cdot 3^{\mathrm{cm}}$ longa, labio postico truncato-ovato $8-9^{\mathrm{mm}}$ longo bidentato, antici IO-I I ${ }^{\mathrm{mm}}$ longi segmentis ovalibus $6^{\mathrm{mm}}$ longis. Stamina medio tubo affixa, antica $17^{\mathrm{mm}}$ longa, posticorum $\mathrm{I} 3^{\mathrm{mm}}$ longorum antheris unilocularibus polleniferis. Discus $\mathrm{I}^{\mathrm{mm}}$ altus, ovario oblongo-ovoideo $4^{\mathrm{mm}}$ longo, stylo capillari $2.7^{\mathrm{cm}}$ longo, ovulis ovalibus $\mathrm{I}^{\mathrm{m}}$ longis. Capsula non adest.--Ceteris speciebus adhuc descriptis, omnibus Brasiliensibus aut Peruvianis, haec bracteis bracteolis calycibus praecipue discrepat.

Casillas, Depart. Santa Rosa, Guatemala, alt. $1300^{\mathrm{m}}$, Jan. 1893 , Heyde et Lux, n. 4382 Pl. Guat. \&c., qu. ed. Donn. Sm.

Hernandia didymantha Donn. Sm.--Ramuli cum foliis petiolisque glaberrimi. Folia oblongo-ovata a medio linea arcuata sursum angustata basi obtusa aut rotundata penninervia. Paniculae folia superantes praeter involucella totae incanae, ramis secundariis bifloris, bracteis 4 oblongis. Flos masculinus solitarius 3-merus, pedicello filiformi. Flos femininus 4-merus, involucello truncato pyramidali subclauso, fructifero sphaerico drupam subaequimagnam arcte includente, semine globoso.

Arbor $15^{\mathrm{m}}$ alta, trunco excelso, ramis inferioribus reflexis, superioribus erecto-patentibus, floribus porraceis (ex schedula et icone photographica Pitterianis). Folia $\mathrm{I} 2-\mathrm{I} 8^{\mathrm{cm}}$ longa $4 \cdot 5-7^{\mathrm{cm}}$ lata tenuiter coriacea, nervis lateralibus 
utrinque $7-8$, petiolis glabris canaliculatis $3-6^{\mathrm{cm}}$ longis. Paniculae circa 5 terminales et ex axillis foliorum superiorum decrescentium prodeuntes cum pedunculo $9-\mathrm{I} \mathrm{I}^{\mathrm{cm}}$ longo adjecto $\mathrm{I}^{\mathrm{I}}-23^{\mathrm{cm}}$ longae corymbiformes densiflorae, ramis secundariis arcuatim adscendentibus, inferioribus $2-3^{\mathrm{cm}}$ longis. Bracteae jam ante anthesin reflexae $8^{\mathrm{mm}}$ longae $3.5^{\mathrm{mm}}$ latae apice rotundatae. Floris masculini pedicellus filiformis $7^{\mathrm{mm}}$ longus, altero anantho brevi rarissime exstante, segmenta 6 oblongo-elliptica $5^{\mathrm{mm}}$ longa obtusa, glandulae 6 liberae subsessiles, antherae oblongae $1.5^{\mathrm{mm}}$ longae filamenta aequantes. Floris feminini pedicellus $\mathrm{I}^{\mathrm{mm}}$ longus, involucellum coriaceum parce pubescens siccitate nigrescens $3^{\mathrm{mm}}$ altum atque latum, ore integro $\mathrm{I}^{\mathrm{mm}}$ lato, segmenta 8 uti glandulae 4 masculinis similia, stylus sursum incrassatus et quadrangularis $4^{\mathrm{mm}}$ longus, stigma obliquum subintegrum nudum. Involucellum fructiferum crasse coriaceum $2.2^{\mathrm{cm}}$-diametrale, drupa $\mathbf{I} .9^{\mathrm{cm}}$-diametralis ecostata (ut videtur).

H. Guianensi Aubl. proxima recedit praesertim foliis penninerviis, flore masculino singulo graciliter pedicellato, involucello jam sub anthesi paene clauso, drupa minore.

Ad litora Atlantica prope Punta Mona, Costa Rica, Sept. I 898, Pittier, n. I 2682 herb. nat. Cost.

Brosimum heteroclitum Donn. Sm.-Scandens. Folia membranacea glabrescentia oblongo-elliptica cuspidato-acuminata basi obtusa aut acutiuscula crenulato-serrulata, stipulis bracteisque a basi lata filiformibus. Receptacula in axillis duabus supremis approximatis solitaria sessilia maxima, masculinorum loculis $\mathrm{I}-3$, ovulis abortivis in quoque loculo pluribus.

Suffrutex in arborum truncis scandens et radicans, ramis virgatis $30-40^{\mathrm{cm}}$ longis glabris. Folia nascentia pubescentia, vetustiora supra punctis minutis albidis scabriuscula costa nervisque pubescentia I O-I $6^{\mathrm{cm}}$ longa $4.5-6.5^{\mathrm{cm}}$ lata, nervis lateralibus utrinque 6-8, venis transversis subparallelis, petiolis $\mathrm{I}-3^{\mathrm{cm}}$ longis sicut stipulae bracteaeque $6^{\mathrm{mm}}$ longae pubescentibus. Receptacula masculina globosa $\mathrm{I}-\mathrm{I} \cdot 3^{\mathrm{cm}}$-diametralia demum ovalia et usque ad $2^{\mathrm{cm}}$ longa crasse corticata ore bracteolis crassis ovatis munita, ovulis abortivis lateraliter affixis. Cetera desunt. Species habitu scandente et ovulis rudimentariis compluribus valde anomala. Receptacula vetustiora staminibus derasis tantum suppetunt.

Jiménez, Llanuras de Santa Clara, Costa Rica, alt. 250 $0^{\mathrm{m}}$, Mart. I896, Donn. Sm., n. 5 I I 7, Pl. Guat., \&c., qu. ed. Donn. Sm.

Pilea ptericlada Donn. Sm.-Dioica. Glabra. Folia oblongoelliptica utrinque acuminata in petiolum brevem cuneato-attenuata 
supra trientem inferiorem grosse crenato-serrata, nervis basilaribus trientem superiorem attingentibus et costa subtus explanatis fuscis, stipulis quam petiolis paulo brevioribus. Pedunculi feminini foliis breviores et cymarum axes alati.

Herbacea. Caulis e basi lignosa radicante assurgens in exemplis obviis 6-I $5^{\mathrm{cm}}$ altus, nodis approximatis. Cujusve paris folia aequalia aequilatera triplinervia $5^{--\mathrm{I}} \mathrm{I}^{\mathrm{cm}}$ longa $3-5^{\mathrm{cm}}$ lata summa apice obtusiuscula supra cystolithis fusiformibus et punctiformibus farcta subtus plerumque nuda, foliis inferioribus minoribus, serraturis obtusis, costa nervisque supra immersis, stipulis orbiculari-ovatis $5-8^{\mathrm{mm}}$ longis binerviis, petiolis $6-1 \mathrm{O}^{\mathrm{mm}}$ longis. Pedunculi $2-6^{\mathrm{cm}}$ longi $2-3^{\mathrm{mm}}$ lati, cymis pyramidatis $2-3^{\mathrm{cm}}$ altis $2.5-3.5^{\mathrm{cm}}$ latis, ramis ramulisque patulis circa $\mathrm{I}^{\mathrm{mm}}$ latis sicut pedunculi cystolithis obsitis. Perianthium sessile fructifer bracteolam cystolithis lineatam subaequans, segmentis parum inaequilongis, achenia ventricoso-ovata $2^{\mathrm{mm}}$ longa perianthium dimidio superante rubro-punctulata et marginata. Planta masculina ignota. Secundum methodum Weddellianam juxta P. elegantem Wedd. locari debetur.

In sylvis udis Atirrensibus, Prov. Cartago, Costa Rica, alt. 600 ${ }^{\mathrm{m}}$, Apr. I896, Donn. Sm. n. 6779 Pl. Guat., \&c., qu. ed. Donn. Sm.; Suerre, Llanuras de Santa Clara, Costa Rica, alt. 300m , Apr. I896, Donn. Sm. n. 6780 Pl. Guat., etc., qu. ed. Donn. Sm.--Eadem planta, ut videtur, sub nn. 172 et I 99 Endres in herb. Kew. exstat.

Costus sanguineus Donn. Sm.- Pallide ferrugineo-villosus. Folia discoloria supra bulboso-pilosa oblanceolato-oblonga acutissime acuminata basi minute rotundata subsessilia. Strobilus lanceolato-ovoideus, bracteis pilosis oblongo-ovatis superne infra apicem carinatis, infimis apice foliaceis. Calyx prima tertia parte acute lobatus. Corolla sanguinea, labello pubescente oblongo apice angustata truncata 5-lobulato, ceteris segmentis anguste lanceolato-oblongis, connectivo triangulari labellum paulo superante. Ovarium pilosum.

Ochreae laciniis scariosis usque ad $2^{\mathrm{cm}}$ longis marginatae saepius reticulato-striatae cum vagina extus villosae et intus glanduloso-punctulatae. Folia subtus sericea et ad costam longe villosa I $^{-22^{\mathrm{cm}}}$ longa supra medium 4.5$6.5^{\mathrm{cm}}$ lata basi $8-10^{\mathrm{mm}}$ lata, nervis omnibus a basi prodeuntibus. Strobilus 7-10 $0^{\mathrm{cm}}$ longus circa $3^{\mathrm{cm}}$ crassus, bracteis coriaceis sanguineis circa $4^{\mathrm{cm}}$ longis 20-2 $3^{\mathrm{mm}}$ latis, bracteola $\mathrm{I}$ pubescente lineari-oblonga $18^{\mathrm{mm}}$ longa $6^{\mathrm{mm}}$ lata acuminata, floribus sub quaque bractea singulis. Calyx campanulatus $8-9^{\mathrm{mm}}$ altus pilosus striatus. Corolla purpureo-coccinea (von Tuerckheim in sched.) 5.5-6 $6^{\mathrm{cm}}$ longa, labello $3.3^{\mathrm{cm}}$ longo $\mathrm{I} \cdot 5^{\mathrm{cm}}$ lato a triente superiore deorsum paulo 
angustato, lobulis oblongis $3^{\mathrm{mm}}$ longis rotundatis, ceteris segmentis $26-28^{\mathrm{mm}}$ longis $7-9^{\mathrm{mm}}$ latis, filamento $9-\mathrm{IO}^{\mathrm{mm}}$ lato, connectivo $7^{\mathrm{mm}}$ longo in caudiculam marginibus revolutam producto, anthera $8^{\mathrm{mm}}$ longa, loculis $\mathrm{I}^{\mathrm{mm}}$ inter se distantibus. Ovarium breviter oblongum $5^{\mathrm{mm}}$ longum, stigmate semilunari $3^{\mathrm{mm}}$ lato ciliolato, appendice dorsali ovali apice retusa. Capsula ignota.-C. spicato $\mathrm{Sw}$. proximus differt praecipue indumento et florum indole atque colore.

Cubilquitz, Depart. Alta Verapaz, Guatemala, alt. 350 Tuerckheim, n. 7686 Pl. Guat. \&c., qu. ed. Donn. Sm.

Ischnosiphon Morlae (Eggers, Bot. Centralbl. 53:307. pl. 2) leiostachya Donn. Sm.-Spicae glabrae, bracteis magis elongatis.-Ceteroquin exempla Centrali-Americana cum specimine typico Ecuadoriensi in herb. Kew. asservato prorsus congruunt.

San Pedro Sula, Depart. Santa Barbara, Honduras, alt. $300^{\mathrm{m}}$, Sept. I 888, C. Thieme, n. 5519 Pl. Guat. \&c., qu. ed. Donn. Sm.; Las Vueltas, Tucurrique, Prov. Cartago, Costa Rica, alt., $635^{\mathrm{m}}$, Dec. I 898 , Tonduz, n. I 2884 herb. nat. Cost.

Calathea dasycarpa Donn. Sm. (§ Eucalathea Koern.)-Folia glabra oblonga 3-4-plo longiora quam latiora in cuspidem gracilem acuminata basi rotundata in articulum cuneato-producta, folio inflorescentiam suffulciente caulinis simili longe petiolato. Pedunculus petiolo brevior bifidus. Spicae pro ratione parvae oblongae interdum basi furcatae, bracteis distichis vix ac ne vix imbricatis dimidiato-ovatis cymbiformibus coriaceis ad margines barbatis, floribus paucis binis. Corollae tubus sepalis 3-plo longior intus villosissimus. Ovarium pilosum. Fructus monospermus.

Folia $60-80^{\mathrm{cm}}$ longa prope medium $\mathrm{I} 8-\mathrm{I} 9^{\mathrm{cm}}$ lata ad costam subtus pubescentia, cuspide lineari $1.5-2.5^{\mathrm{cm}}$ longa, petiolis cum articulo glabro $5^{-6^{\mathrm{cm}}}$ longa adjecto $43^{\mathrm{em}}$ longis pilosiusculis. Pedunculus $2 \mathrm{I}^{\mathrm{cm}}$ longus basi bractea $\mathrm{I}^{\mathrm{cm}}$ longa apice bractea $3.5^{\mathrm{cm}}$ longa suffultus, ramis $3-5^{\mathrm{cm}}$ longis. Spicae $6-9^{\mathrm{cm}}$ longae, rachis internodiis inferoribus $10-18^{\mathrm{mm}}$ longis, bracteis $10-12$ demum late patulis circa $2^{\mathrm{cn}}$ longis $9^{\mathrm{mn}}$ latis, bracteolis 2 late oblongis Io$14^{\mathrm{mm}}$ longis, exteriore bicarinata et dentibus rotundatis 3 -lobulata, interiore minore unicostata integra, floribus plerumque 4 minute pedicellatis. Sepala lanceolata $7-9^{\mathrm{mm}}$ longa. Corollae pallide luteae (Pittier in sched.) tubus $28^{\mathrm{mm}}$ longus intus valde pluri-costatus. Ovarium obovatum $3^{\mathrm{mm}}$ longum dense longeque adpresso-pilosum, ovulo abortu unico triquetro. Fructus ovalis $\mathrm{IO}^{\mathrm{mm}}$ longus $5^{\mathrm{mm}}$ latus pilosus, pericarpio membranaceo nervoso, semine 
atro-purpureo oblongo $4.5^{\mathrm{mm}}$ longo corrugato. Corolla cum androecio marcida tantum suppetit.

In sylvis ad oras Rio Hondo prope Madre de Dios, Comarca de Limón, Costa Rica, alt. 200m, Nov. 1896, Pittier, n. I0350 herb. nat. Cost.; Agua Buena, Cañas Gordas, Comarca de Limón, Costa Rica, alt. I Ioo ${ }^{\mathrm{m}}$, Feb. I897, Pittier, n. I I 36 herb. nat. Cost.

Calathea lasiostachya Donn. Sm. (§ Eucalathea Koern.) Folia glabra elongato-ovata apice acuminata inferne rotundata ima basi cuneata, folio inflorescentiam suffulciente conformi dimidio minore petiolis usque ad articulum vaginatis bis longiore. Pedunculi 3-ni petiolum subaequantes. Spicae elongato-oblongae sordide villosae, bracteis stricte distichis late patentibus conduplicatis membranaceis. Ovarium glabrum. Fructus dispermus.

Caulis robustus elatus. Articuli cum vaginis pedunculisque pilosiusculi. Folia $86^{\mathrm{cm}}$ longa $23^{\mathrm{cm}}$ lata a triente inferiore sursum angustata, articulis $9^{\mathrm{cm}}$ longis, petiolis longissimis, vaginis scabriusculis. Folium sub inflorescentia $40^{\mathrm{cm}}$ longum $\mathrm{I} 5^{\mathrm{cm}}$ latum, petiolo cum articulo $3^{\mathrm{cm}}$ longo adjecto $2 \mathrm{I}^{\mathrm{cm}}$ longo. Pedunculi $15-22^{\mathrm{cm}}$ longi ad apicem versus villosi. Spicae II.5-I $7^{\mathrm{cm}}$ longae 5.5-6 ${ }^{\mathrm{cm}}$ latae, lateribus subparallelis. Bracteae 22-32 sublaxe imbricatae dimidiato-ellipticae subfalcatae $3.5^{\mathrm{cm}}$ longae $\mathrm{I} .5^{\mathrm{cm}}$ latae intus sericeae, omnes conformes, bracteolis lineari lanceolatis et linearibus circa $2^{\mathrm{cm}}$ longis. Ovarium nitido-atrum oblongum $6^{\mathrm{mm}}$ longum $4^{\mathrm{mm}}$ latum 3 -loculare abortu 2-ovulatum. Fructus laevis pallidus ellipticus $\mathrm{I}^{\mathrm{mm}}$ longus $6^{\mathrm{mm}}$ crassus, pericarpio pergameneo intus valde reticulato, valvis 3 inaequilatis, minoribus latere septatis, seminibus atris $8^{\mathrm{mm}}$ longis arillosis. Perianthium deficit.

In sylvis ad oras Rio Hondo prope Madre de Dios, Costa Rica, alt. 200"m, Nov. I896, Pittier, n. I0344 herb. nat. Cost.

Calathea Verapax Donn. Sm. (§ Monostiche Koern.)-Glabra. Acaulis. Folia inaequilatera elliptica vel ovato-elliptica acuminata ima basi in articulum brevem producta petiolis subaequilonga. Scapus nudus petiolum superans usque ad medium bractea radicali inclusus basi petiolo et bracteis cinctus. Spica obovato-elliptica, bracteis membranaceis coloratis numerosissimis lanceolatis filiformi-attenuatis subaequilongis. Corollae tubus sepalis linearibus dimidio longior, lobi lanceolati.

Rhizoma bracteas $7-20^{\mathrm{cm}}$ longas apice denticulata petiolos 2 scapumque involventes emittens, petiolo altero toto fere vaginato alterum nudum 
includente. Folia $20-30^{\mathrm{cm}}$ longa $9.5-14^{\mathrm{cm}}$ lata, petiolis cum articulo $\mathrm{I}-2^{\mathrm{cm}}$ longo adjecto $29-33^{\mathrm{cm}}$ longis. Scapus $35-55^{\mathrm{cm}}$ longus. Spica $8-12^{\mathrm{cm}}$ longa circa $4^{\mathrm{cm}}$ lata, rhachi $5^{\mathrm{cm}}$ longa, bracteis in sicco rubescentibus erecto-patentibus, infimis $6.5^{\mathrm{cm}}$ longis $\mathrm{I} .8^{\mathrm{cm}}$ latis, supremis $6^{\mathrm{cm}}$ longis $9^{\mathrm{mm}}$ latis, bracteolis 3 late oblongis $1.7^{\mathrm{cm}}$ longis, exteriore valde bicarinata, floribus $2-4$-nis. Sepala $2 . \mathrm{I}^{\mathrm{cm}}$ longa $2.5^{\mathrm{mm}}$ lata. Corollae coccineae (ut videtur), tubus $3^{\mathrm{cm}}$ longus, lobi $\mathrm{I} .5^{\mathrm{cm}}$ longi $5^{\mathrm{mm}}$ lati acuti. Androecii labellum $\mathrm{I}^{\mathrm{cm}}$ longum $7^{\mathrm{mm}}$ latum, lobus cucullatus appendice filiformi $4^{\mathrm{mm}}$ longa instructus, staminifer anthera $2.5^{\mathrm{mm}}$ longa superatus. Stylus incurvus, stigmate valde inflexo. Ovarium glabrum oblongo-obovatum 3-loculare. Fructus non adest.-Ad C. Petersenii Eggers habitu accedens spica et florum fabrica recedit.

Rubelcruz, Depart. Alta Verapaz, Guatemala, alt. Iоoо ${ }^{\mathrm{m}}$, Maj. I 887, von Tuerckheim, n. I269 P1. Guat. \&c., qu. ed. Donn. Sm.; Sacolal, Depart.

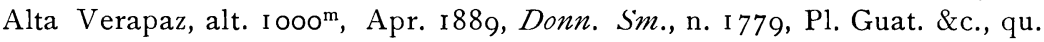
ed. Donn. Sm.

Callisia grandiflora Donn. Sm.- Folia a basi rotundata sessili elliptico-lanceolata. Cyma elongata cincinnalis, pedicellis compluribus, floribus 3-meris maximis. Stamina fertilia 3, filamentis sursum barbatis, loculis divergentibus connectivo amplissimo brevioribus. Stamina sterilia 3 nuda. Capsula 3-valvis, loculis monospermis.

Praeter ocreas ciliatas glabra. Folia 6-I $2^{\mathrm{cm}}$ longa $\mathrm{I} \cdot 5^{-2} \cdot 5^{\mathrm{cm}}$ lata acutissima. Cyma paniculiformis 6-I ${ }^{\mathrm{cm}}$ longa, rhachi flexuosa, axibus primariis I. $5-2.5^{\mathrm{cm}}$ longis, lateralibus $7-20^{\mathrm{mm}}$ longis, bracteis inferioribus foliaceis $\mathrm{I}-5^{\mathrm{cm}}$ longis, superioribus depauperatis sicut bracteolae minimae subspathaceis, pedicellis circa $5-9$-fasciculatis $7-\mathrm{I}^{\mathrm{mm}}$ longis. Sepala herbacea albopunctata oblongo-elliptica $6.5^{\mathrm{mm}}$ longa $2.5^{\mathrm{mm}}$ lata sub anthesi reflexa. Petala ex schedula Tuerckheimiana alba in sicc. pallide flavescentia oblongoelliptica $9^{\mathrm{mm}}$ longa $4^{\mathrm{mm}}$ lata obtusa. Stamina fertilia $6-7^{\mathrm{mm}}$ longa, pilis aureis longissimis densissimis, antheris cinnabarinis transversim ovalibus $\mathrm{I} .5^{\mathrm{mm}}$ latis utrinque praesertim apice bifidis, loculis oblongis $0.5^{\mathrm{mm}}$ longis subdivaricatis. Stamina imperfecta $2-3^{\mathrm{mm}}$ longa, loculis connectivo parvulo subdiscretis. Ovarium ovale $\mathrm{I}^{\mathrm{mm}}$ longum stylum aequans, stigmate capitellato papilloso. Capsula ovalis $5-6^{\mathrm{mm}}$ longa abortu saepius bisperma, seminibus erubescentibus rugulosis elongato-oblongis $4{ }^{\mathrm{mm}}$ longis.-Species staminibus et perfectis et rudimentariis abnormalis ad Tradescantiae sect. Descantariam accedens.

In dumetis et frutectis prope Cubilquitz, Depart. Alta Verapaz, Guatemala, alt. $350^{\mathrm{m}}$, Mart. I900, H. von Tuerckheim, n. 7684 Pl. Guat. \&c., qu. ed. Donn. Sm.

Baltimore, MD. 